\title{
PROPUESTA DE PROTOCOLIZACIÓN PARA LA PRÁCTICA DE LA TERAPIA ELECTROCONVULSIVA EN EL HOSPITAL DE CLÍNICAS DE LA UNIVERSIDAD NACIONAL DE ASUNCIÓN, PARAGUAY
}

\author{
PROTOCOL'S PROPOSAL FOR THE PRACTICE OF \\ ELECTROCONVULSIVE THERAPY AT HOSPITAL DE CLÍNICAS \\ OF THE NATIONAL UNIVERSITY OF ASUNCIÓN, PARAGUAY
}

\section{María Emilia CHÁVEZ¹.}

${ }^{1}$ Auxiliar de la Enseñanza de Psiquiatría, Facultad de Ciencias Médicas, Universidad Nacional de Asunción, San Lorenzo - Paraguay.

Cómo citar este artículo: Chávez ME. Propuesta de protocolización para la práctica de la terapia electroconvulsiva en el Hospital de Clínicas de la Universidad Nacional de Asunción, Paraguay. Medicina Clínica y Social. 2017;1(2):126-142.

\section{RESUMEN}

La electroconvulsión básicamente consiste en provocar una crisis comicial generalizada de una determinada duración, mediante la aplicación de energía eléctrica sobre un paciente anestesiado, con una finalidad terapéutica. La mayoría de las nuevas teorías se enfocan en los cambios a nivel de neurotransmisores. Numerosos estudios investigan los cambios biológicos que produce la terapia electroconvulsiva, pero a causa de las graves dificultades metodológicas a las que deben enfrentarse no se ha podido concluir con exactitud cuáles de estos cambios son los responsables de la acción terapéutica. La terapia electroconvulsiva ha demostrado gran efectividad donde otros procedimientos han fallado. Inicialmente, la indicación principal era la esquizofrenia. En la actualidad continúa siendo la terapia de elección para el tratamiento de una gran variedad de enfermedades mentales, pero con mayor énfasis en los trastornos afectivos. La unidad de terapia electroconvulsiva se debe ubicar físicamente en el Hospital de Clínicas y depender del Servicio de Psiquiatría del citado hospital, debe constar de una sala de espera, una sala de tratamiento y una sala de recuperación, las cuales deben estar dotadas de los materiales y fármacos suficientes para inducir la anestesia, monitorizar las funciones fisiológicas y garantizar la ventilación y reanimación. Este artículo tiene por objetivo presentar una propuesta de protocolización de la práctica de la terapia electroconvulsiva en el Hospital de Clínicas de la Universidad Nacional de Asunción, Paraguay.

Palabras clave: Terapia electroconvulsiva; Psiquiatría; Protocolización; Paraguay.

\section{ABSTRACT}

Electroconvulsive therapy basically consists in generate a generalized epileptic seizure of a certain duration, by applying power on an anesthetized patient, for therapeutic purposes. Most new theories focus on changes at the level of neurotransmitters. Numerous studies investigate the biological changes that produces the electroconvulsive therapy, but because of serious methodological difficulties, they could not conclude exactly what of these changes are responsible for the therapeutic action. Electroconvulsive therapy has proven very effective where other methods have failed. Initially, the main indication was schizophrenia. Today it remains as the therapy of choice for the treatment of a variety of mental illnesses, but with greater emphasis on affective disorders. The electroconvulsive therapy unit should be physically placed at the Hospital de Clínicas and rely on the Department of Psychiatry of that hospital. 
It must consist of a waiting room, a treatment room and a recovery room, which should be provided with the materials and sufficient drugs to induce anesthesia, to monitor physiological functions and ensure ventilation and resuscitation. This article aims to present a protocol's proposal for the practice of electroconvulsive therapy at the Hospital de Clínicas of the National University of Asunción, Paraguay.

Keywords: Electroconvulsive therapy; Psychiatry; Protocol; Paraguay.

\section{INTRODUCCIÓN}

La terapia electroconvulsiva (TEC) es el tratamiento biológico más antiguo que se encuentra todavía disponible en la práctica psiquiátrica y sigue siendo uno de los principales tratamientos para el abordaje de enfermedades mentales severas $(1,2)$.

La TEC ha sido siempre vista como un procedimiento controvertido. En la década de los 70s con la aparición del movimiento antipsiquiatra, la TEC fue vista como un método represivo y deshumanizador, correlacionándola como una herramienta más para la represión y la tortura.

La TEC fue objeto prioritario de las críticas de la antipsiquiatría, que influyó en la opinión pública, en los medios de comunicación e incluso en un sector importante de médicos y otros trabajadores de la salud.

La técnica de la TEC ha evolucionado de manera importante a lo largo de los años. Actualmente se requiere control anestésico, miorrelajación, ventilación artificial y monitorización con electrocardiograma y electroencefalograma. Estos elementos, logran una mayor precisión en la intensidad de la estimulación eléctrica y disminución de los efectos adversos y complicaciones asociadas al tratamiento.

Actualmente, no cabe ninguna duda que la implementación de la TEC en los servicios de Psiquiatría en todo el mundo se hace imprescindible.

Las indicaciones para la aplicación de la TEC se encuentran bien documentadas y fundamentadas, los efectos adversos están debidamente caracterizados y en la mayoría de los países existe protocolización de su uso, avalado por opiniones expertas de psiquiatras, anestesiólogos y otros profesionales.

La protocolización de la TEC tiene como finalidad iniciar la práctica de este procedimiento como una alternativa válida, factible y accesible a la población de pacientes que consultan en la Cátedra y Servicio de Psiquiatría del Hospital de Clínicas, con los beneficios terapéuticos, académicos, de formación e investigación, que esta práctica representaría.

\section{CONCEPTO DE TEC}

La electroconvulsión básicamente consiste en provocar una crisis comicial (convulsión tónicoclónica) generalizada y de una determinada duración, mediante la aplicación de energía eléctrica, sobre un paciente anestesiado, con una finalidad terapéutica (2).

La TEC se puede definir como el conjunto de electroconvulsiones para completar un tratamiento (el número de electroconvulsiones es variable dependiendo de la patología y de las peculiaridades de cada paciente) (2). 


\section{MECANISMO DE ACCIÓN}

Uno de los puntos que se blanden contra la TEC es el desconocimiento de su mecanismo de acción, tanto en la producción de su efecto terapéutico como a nivel de la aparición de efectos adversos (2).

Al intentar brindar una explicación sobre su fisiopatología, se han formulado distintas hipótesis, basadas en hallazgos posteriores al procedimiento.

\section{Teoría de la convulsión generalizada}

Esta teoría se ha fundamentado en estudios de Ottoson. En ellos se ha replicado como la TEC activa regiones anatómicas solo a través de estímulos que superen el umbral convulsivo. Para que la TEC ejerza sus propiedades beneficiosas se debe inducir una convulsión bilateral generalizada $(3,4)$. De cualquier manera, la convulsión es necesaria para explicar la eficacia de la TEC pero no es suficiente.

\section{Teoría neuroendócrina diencefálica}

En la melancolía se pierde el balance neuroquímico cerebral y que la TEC la restablece. Habría un disbalance en el eje Hipotalámo-Hipófisis-Adrenal aumentando la secreción de cortisol (4).

\section{Teoría de los neurotransmisores}

Estudios en animales han demostrado que existe un aumento en la síntesis y el turn-over de serotonina $(5 \mathrm{HT})$ inmediatamente después de las crisis. La TEC sensibiliza a los receptores 5 HT1A post sinápticos sin alterar estos receptores en las neuronas presinápticas (5). Estudios de Tomografía por Emisión de Positrones demostraron que la TEC disminuye la concentración de receptores $5 \mathrm{HT} 2$, especialmente en el pre-frontal y sistema límbico y se correlaciona con disminución de puntajes e las escalas HAM-D (4).

Dentro de los mecanismos que se asocian a los síntomas depresivos, se ha hallado mayor bloqueo de estímulos $\alpha 2$ adrenérgicos del locus coeruleus, mejoría en la expresión de receptores D1 y D3 a nivel estriatal $(6,7)$ sin cambios en receptores D2 (5).

\section{Flujo sanguíneo cerebral y TEC}

La mayoría de las nuevas teorías se enfocan en los cambios a nivel de neurotransmisores. Este hecho no implica que los cambios macroestructurales no sean relevantes.

Las sesiones de electroconvulsión aumentan el riego sanguíneo a nivel de ganglios basales, amígdala, cerebelo, tálamo, hipotálamo y zonas inferiores de los lóbulos frontales, parietales y occipitales (4).

Numerosos estudios investigan los cambios biológicos que produce la TEC, pero a causa de las graves dificultades metodológicas a las que deben enfrentarse no se ha podido concluir con exactitud cuáles de estos cambios son los responsables de la acción terapéutica. Las diferentes teorías neurobiológicas no pueden ser contempladas de forma independiente debido a la complejidad de la función cerebral. Es por esto que la TEC sigue siendo un tratamiento "empírico" cuya utilización queda plenamente justificada por su eficacia terapéutica. 


\section{INDICACIONES CLÍNICAS GENERALES}

La TEC ha demostrado gran efectividad donde otros procedimientos han fallado. Inicialmente, la indicación principal era la esquizofrenia. En la actualidad continúa siendo la terapia de elección para el tratamiento de una gran variedad de enfermedades mentales pero con mayor énfasis en los trastornos afectivos.

La población general, incluso muchos médicos, experimentan temor y reserva ante la aplicación de la TEC. Esto se debe en gran medida a que la TEC, antecede al desarrollo de la psicofarmacología y otras terapias, por lo que se la utilizó de forma indiscriminada para tratar condiciones clínicas que no justificaban su utilización, como la ludopatía, las fobias, las conversiones, las adicciones y la homosexualidad.

La decisión de aplicar TEC se basa en los criterios clínicos de gravedad, la consideración de indicaciones y contraindicaciones, la resistencia a otro tipo de tratamiento, la valoración de situaciones especiales como el embarazo y el alto riesgo de suicidio, antecedentes de buena respuesta y preferencia del paciente (1).

La tabla 1 resume las indicaciones clínicas generales en las que se recomienda el uso de TEC como primera opción, incluso antes del tratamiento farmacológico (8).

\section{TABLA 1. INDICACIONES CLÍNICAS GENERALES DE LA TEC}

1. Cuando se requiere una mejoría rápida y definitiva a raíz de la gravedad de la enfermedad psiquiátrica, cuando existe una intensa agitación, estupor catatónico o gran desorganización conductual.

2. Cuando los riesgos de la TEC son menores en comparación a otros tratamientos.

3. Si la historia previa refiere antecedentes de una pobre o nula respuesta farmacológica o de buena respuesta previa a la TEC.

4. Si aparecen graves efectos adversos o intolerancia a la farmacoterapia.

5. Preferencia del paciente.

\section{INDICACIONES CLÍNICAS PRIMARIAS}

\section{Episodio depresivo mayor (unipolar o bipolar)}

El diagnóstico de trastorno depresivo mayor (TDM) es la primera indicación para aplicación de la TEC, especialmente si la depresión se asocia a un alto riesgo suicida, la inanición, la inhibición o agitación psicomotora severa y TDM con síntomas psicóticos $(3,8)$. Aunque en los cuadros agudos la eficacia es similar a la de los antipsicóticos, las depresiones psicóticas tratadas con TEC parecen tener cifras menores de recaídas $(1,9)$.

Son predictores de buena respuesta la existencia de ideas delirantes, comienzo brusco, duración inferior a un año, inanición, despertares precoces, pérdida de peso, antecedentes familiares de depresión. La TEC es más eficaz cuanto más sean los signos de endogenicidad del cuadro depresivo (1).

Como indicación alterna de la depresión mayor, se ha considerado que la TEC puede ser útil en pacientes que hayan tenido fallo terapéutico de dos antidepresivos por un periodo no menor de las 6 semanas, sin embargo, a largo plazo la eficacia de la TEC y los antidepresivos es similar o inferior, por lo que debe continuarse el tratamiento con TEC de mantenimiento o 
fármacos. También la TEC se ha mostrado eficaz cuando existe contraindicación de antidepresivos y en la depresión en el embarazo (1).

\section{Episodio maniaco}

La TEC es, como mínimo, tan eficaz como el litio (3), aunque la respuesta es más rápida (1). Se considera tratamiento de elección para manías, especialmente aquellas refractarias al tratamiento psicofarmacológico, la mujer embarazada, cicladores rápidos, episodios mixtos y manías asociadas con alto riesgo de suicidio y agitación (1-3).

\section{Esquizofrenia y otras psicosis}

La TEC ganó su status terapéutico gracias a su efectividad en la esquizofrenia, aunque con la aparición de nuevos antipsicóticos, la misma ha ido cayendo en desuso. Pese a lo anterior, la TEC sigue siendo de elección para la esquizofrenia refractaria (10).

La TEC es un tratamiento eficaz en casos de clínica psicótica aguda, no así en la esquizofrenia crónica, y es claramente más efectiva para los síntomas positivos y afectivos. Si predomina la clínica cognitiva o negativa no obtienen buena respuesta $(1,11)$. Otras indicaciones son la catatonía, desorganización grave con negativa a ingesta de líquidos y sólidos y como coadyuvante para el tratamiento de los síntomas depresivos especialmente si hay riesgo de suicidio (12).

Para las guías del National Institute of Clinical Excellence (NICE), la TEC es indicación primaria en la catatonía y en pacientes que clínicamente muestren signos de catatonía con refractariedad al tratamiento con benzodiacepinas (13) y como un coadyuvante de la clozapina en pacientes refractarios al tratamiento (14).

En países subdesarrollados, donde la disponibilidad y el acceso a la medicación antipsicótica atípica son muy limitados, se recomienda la utilización de la TEC debido a su bajo coste, accesibilidad y sus efectos a corto plazo (10).

Es importante recordar que bajo ningún modo puede considerarse a la TEC como sustituto del tratamiento psicofarmacológico, más bien como terapia adyuvante (10).

\section{Otras condiciones psiquiátricas}

La TEC en la distimia, los trastornos de la conducta alimentaria, trastornos por consumo de sustancias y los trastornos de la personalidad, no posee evidencia suficiente que sustente estas indicaciones (15).

\section{INDICACIONES CLÍNICAS SECUNDARIAS}

\section{Epilepsia}

La TEC puede ser considerada en pacientes con epilepsias refractarias al tratamiento farmacológico. Se debe tener en cuenta que la medicación anticonvulsivante dificulta que la TEC pueda inducir convulsiones, por lo que las dosis deben minimizarse y monitorizar los niveles sanguíneos de anticonvulsivantes antes de iniciar las electroconvulsiones $(16,17)$. 


\section{Enfermedad de Parkinson}

La TEC puede resultar beneficiosa en fenómenos de On-Off. Se deben incluir sólo pacientes refractarios a la medicación dopaminérgica y reducir la dosis de los agentes dopaminérgicos a la mitad luego de la TEC a fin de prevenir posibles cuadros de delirium post-TEC y disquinesias (18).

\section{Síndrome neuroléptico maligno}

Se recomienda no utilizar como agente anestésico la succinilcolina por el riesgo de empeoramiento de la hipertermia (18).

\section{CONTRAINDICACIONES DE LA TEC}

No existen contraindicaciones médicas absolutas. En caso de enfermedad médica no psiquiátrica asociada, se debe considerar consulta con el especialista y ajustar el tratamiento si fuera necesario (16). Se debe valorar: 1 . El posible riesgo asociado al tratamiento; y, 2. Riesgo en caso de no realizarse el tratamiento y el beneficio buscado. En cuanto a las contraindicaciones relativas, la tabla 2 presenta la principales.

\section{TABLA 2. CONTRAINDICACIONES RELATIVAS DE LA TEC}

\begin{tabular}{|c|l|}
\hline $\begin{array}{c}\text { Patología cardiovascular } \\
\text { grave }\end{array}$ & $\begin{array}{c}\text { Infarto agudo de miocardio reciente, cardiopatía isquémica e hipertensión } \\
\text { arterial grave y refractaria. Utilización de marcapasos. Terapia anticoagu- } \\
\text { lante. Enfermedades tromboembólicas y tromboflebitis. }\end{array}$ \\
\hline $\begin{array}{c}\text { Patología cerebral } \\
\text { Riesgo de hemorragia }\end{array}$ & $\begin{array}{c}\text { Aumento de la presión intracraneal. Procesos intracraneales ocupantes de } \\
\text { espacio. Traumatismo craneoencefálico. Hidrocefalia. }\end{array}$ \\
\hline $\begin{array}{c}\text { Procesos degenerativos } \\
\text { óseos }\end{array}$ & Oneurisma y malformaciones vasculares. \\
\hline Alteraciones endocrinas & Hipertiroidismo, feocromocitoma, diabetes mellitus. \\
\hline Riesgo anestésico & ASA superior a 3. \\
\hline
\end{tabular}

\section{POBLACIONES ESPECIALES}

\section{Niños y adolescentes}

Los estudios en población infantil y adolescente todavía son insuficientes para emitir una recomendación clara en la utilización de la TEC, no obstante tampoco existen contraindicaciones significativas (15). 
La Academia Americana de la Niñez y Adolescencia recomienda que la TEC solo sea utilizada bajo criterios diagnósticos de depresión mayor con o sin síntomas psicóticos, episodios maniacos, trastorno esquizoafectivo, esquizofrenia o certeza en el diagnóstico de síndrome neuroléptico maligno. Asimismo, puede ser utilizada en condiciones clínicas como la suicidabilidad, psicosis y manía refractaria o reticencia a comer.

No son indicaciones validadas, los trastornos de la conducta alimentaria, los trastornos de la personalidad, los trastornos de la conducta, el trastorno por déficit de atención con hiperactividad, el trastorno de estrés post traumático ni el manejo conductual en pacientes con retardo mental (19).

\section{Embarazo}

La TEC en el embarazo se considera un procedimiento relativamente seguro pero no inocuo. La mortalidad de TEC en embarazadas da cifras similares al dar TEC en el resto de la población. Se puede aplicar en los tres trimestres del embarazo. El riesgo de trasmisión del estímulo eléctrico al feto o la transmisión de factores teratogénicos es mucho menor a los aportados por los fármacos. Se recomienda que sea el obstetra quien dé el visto bueno para el procedimiento y que el mismo tenga monitorización fetal una hora antes y después de cada procedimiento (20).

\section{Adultos mayores}

La utilización de TEC en este grupo poblacional ha aumentado en los últimos años, teniendo en cuenta el incremento del envejecimiento poblacional y mayor sobrevida de los pacientes psiquiátricos. Esta población presenta mayor número de aspectos clínicos a tener en cuenta en el momento de la prescripción de un antidepresivo y las depresiones llamadas "vasculares" tienen mala respuesta terapéutica a la medicación.

La TEC también se ha considerado de gran utilidad cuando existe comorbilidad con la depresión con la enfermedad de Parkinson y con la demencia (21). Los pacientes con deterioro cognitivo de base pueden tener. Se debe tener en cuenta que en muchos casos la intensidad de los estímulos debe ser mayor, porque el umbral convulsivo puede aumentar con la edad (20).

\section{MEDICAMENTOS Y TEC}

Los pacientes que reciben benzodiazepinas deben discontinuar su uso, de forma progresiva, debido a que las mismas inhiben la aparición de convulsiones y por ende el efecto clínico de la TEC (18). Las benzodiacepinas de acción larga no deben ser suspendidas pues existe el riesgo de disminuir el umbral convulsivo, luego de haber sido establecido.

Si se requiere sedación los días previos, se recomienda utilizar antipsicóticos de baja potencia. Si el paciente toma nitratos o antiarrítmicos los mismos deben de seguirse usando antes y durante el ciclo de TEC (20). 
La TEC eleva la presión arterial de modo transitorio y algunos pacientes experimentan hipotensiones posteriores, por lo que la Asociación Americana de Psiquiatría recomienda no utilizar antihipertensivos previo a la TEC ya que podrían provocar eventos vasculares isquémicos (20).

Para pacientes asmáticos, se recomienda dar broncodilatadores $\beta$-agonistas una hora previa a cada procedimiento. La teofilina está absolutamente contraindicada dado el alto riesgo de desarrollar un status epiléptico (20).

La indicación de la TEC en la mayoría de las guías advierte sobre su uso en combinación con antidepresivos por el aumento de los efectos adversos cognitivos $(22,23)$. Por este motivo no se recomienda su instauración si no se estaba utilizado medicación previamente, tampoco es recomendable la suspensión brusca (22).

El uso de antidepresivos funciona mejor durante la fase de mantenimiento y para evitar recaídas post TEC.

La clozapina no debe utilizarse 12 horas previas a la TEC por riesgo de cardiotoxicidad y se debe utilizar menores dosis de corriente eléctrica por el riesgo de disminución del umbral convulsivo (20).

No se contraindica la administración de litio, no obstante, debe disminuirse el estímulo de la corriente.

\section{ORGANIZACIÓN Y FUNCIONAMIENTO DE LA UNIDAD DE TEC}

\section{La unidad de TEC}

La unidad de TEC se debe ubicar físicamente en el Hospital de Clínicas y depender del Servicio de Psiquiatría del citado hospital, debe constar de una sala de espera, una sala de tratamiento y una sala de recuperación, las cuales deben estar dotadas de los materiales y fármacos suficientes para inducir la anestesia, monitorizar las funciones fisiológicas y garantizar la ventilación y reanimación (16).

La unidad debe poseer ser salas bien iluminadas y ventiladas, con separación física entre las diferentes salas (20).

\section{Sala de TEC}

La sala de TEC debe disponer como mínimo de una máquina de TEC de pulso breve, una camilla de base sólida, oxígeno, succión, Unidad de monitoreo cardiaco y equipamiento de paro cardiaco.

\section{Sala de recuperación}


Es una sala que se utilizará para manejar a los pacientes post TEC, donde se debe vigilar de forma estricta la vía aérea y la toma de signos vitales cada 15 minutos. El tiempo de recuperación oscila entre los 5 a los 30 minutos. Para abandonar la zona de TEC el paciente debe encontrarse despierto, responder órdenes simples y signos vitales en rango $(1,17)$.

El paciente debe permanecer al menos 4 horas en el centro y se debe interrogar sobre dolores musculares, cefaleas y náuseas (1).

\section{El comité de TEC}

Estará integrado por dos médicos psiquiatras, un anestesiólogo, un médico internista, un profesional de enfermería (17).

\section{Actividades}

La actividad de la Unidad se orientará en cuatro vertientes: terapéutica, docente/formación, investigación y administrativa. El comité de TEC es el encargado de proponer y de revisar periódicamente el protocolo de la TEC y deberá analizar si los casos propuestos para la realización de la TEC cumplen las indicaciones establecidas para la realización de dicho tratamiento.

\section{DOCUMENTACIÓN CLÍNICA}

\section{La historia clínica psiquiátrica}

La historia clínica psiquiátrica debe ser una evaluación longitudinal que informe sobre la situación clínica del paciente y que permita valorar con mayor exactitud la posible respuesta del paciente y la aparición de efectos secundarios. Se deben consignar los hábitos tóxicos, alergias medicamentosas, tratamientos farmacológicos actuales y anteriores. Antecedentes de respuesta terapéutica previa.

Establecer la necesidad de TEC y reflejarlo en la historia. Evaluación de situación de riesgo.

Se deben incluir los datos suficientes para establecer la situación basal del paciente que facilite el seguimiento clínico del tratamiento con una detallada exploración psicopatológica y escalas que correspondan a cada caso.

\section{Evaluación clínica por el comité de TEC}

El comité de TEC debe evaluar si el paciente cumple criterios psiquiátricos y clínicos para la realización de la TEC. Plantea posibles complicaciones que podría presentar el paciente y determinar si el beneficio supera los riesgos que conlleva el uso de la TEC como tratamiento.

\section{Evaluación clínica, pre-anestésica y enfermería}

El paciente debe contar con una evaluación clínica completa, análisis laboratoriales actualizados, electrocardiograma, radiografía de tórax, estudio de condiciones médicas concomitantes. En algunos casos será necesario realizar interconsultas con cardiología, neurología o algún otro departamento. 
Se deberá establecer claramente la línea de acción en cuanto a la utilización de medicación no psiquiátrica previo a la TEC. El personal de enfermería debe llevar igualmente un registro de todas sus actividades y cualquier efecto que pueda ver en el paciente post TEC.

\section{BASE LEGAL Y ÉTICA}

\section{Consentimiento informado}

La relación médico-paciente es bilateral, y cómo todas las de esta índole, determina derechos y deberes recíprocos tanto éticos como estrictamente jurídicos (23). La función del consentimiento, no es simplemente obtener la firma del paciente, sino que el elemento más importante es asegurarse que el paciente reciba información completa y adecuada, de forma que pueda tomar una decisión $(16,24)$.

La información debe darse tanto de forma oral como escrita $(16,25)$. El consentimiento debe incluir el nombre del paciente y del médico, nombre del procedimiento a realizar, información sobre las complicaciones frecuentes, la declaración del paciente de que se le ha explicado los riesgos personalizados, que conoce que en cualquier momento puede revocar el consentimiento y satisfacción con la información y las aclaraciones recibidas, fecha y firma del médico (18).

Con relación a las personas que no son capaces de consentir, como por ejemplo los niños, sus padres o representantes legales podrán otorgar su consentimiento; de cualquier manera los niños, si tienen edad suficiente, deberán recibir toda la información concerniente al procedimiento. Es legalmente permitido que se practique la TEC a pacientes que no lo deseen, en el caso de las personas cuya situación psicopatológica, no le permita dar su consentimiento (por ejemplo, episodios psicóticos, enfermedad de Alzheimer), sus familiares directos o responsables de su cuidado, podrán dar su consentimiento. Si no existen responsables para el procedimiento, el Estado se hace responsable, bajo la premisa que el procedimiento se es dado de emergencia para buscar una mejoría del estado de salud $(18,20,24)$. En todas estas condiciones debe primar el principio de beneficencia (24).

El consentimiento debe ser recogido por un médico que tenga pleno conocimiento de la TEC (18). El consentimiento informado por anestesia se puede realizar de forma conjunta o independiente al consentimiento de la TEC. Otras de las funciones del consentimiento informado es proteger legalmente a los profesionales de posibles denuncias por información insuficiente a los pacientes (24). El consentimiento debe obtenerse antes de iniciar la TEC (25). El paciente tiene la libertad de rechazar el tratamiento incluso luego de haber otorgado el consentimiento.

En caso de pacientes que requieren el régimen de TEC de mantenimiento el consentimiento debe ratificarse como mínimo cada 6 meses (16).

\section{PROCEDIMIENTO DE LA TEC}

Es importante que, antes de la primera sesión, el psiquiatra compruebe que todos los estudios pre-operatorios estén completos y que se el paciente haya recibido la información y autorizado el procedimiento, explicitado en el consentimiento informado (16). 


\section{Evaluación pre-TEC}

La tabla 3 presenta recomendaciones de la Asociación Americana de Psiquiatría y otras organizaciones $(2,16,28,20)$.

\section{TABLA 3. EVALUACIÓN PRE-TEC}

1. Elaboración de historia clínica completa.

2. La justificación de la aplicación de la TEC.

3. Evaluación médica y física que determine el biotipo del paciente. Se debe realizar evaluación de la cavidad oral y la dentadura.

4. Evaluación anestesiológica completa que incluya recomendaciones sobre cambios para evitar problemas específicos con la medicación.

5. Analítica Básica, hemograma, urea, creatinina, NA+, K+y pruebas de coagulación.

6. Electrocardiograma, radiografía de tórax.

7. Valoración del estado cognitivo del paciente.

\section{Aplicación de la TEC: el aparato de TEC}

Los aparatos de TEC más modernos incorporan pulsos breves de onda cuadrada que, al provocar convulsión a una dosis menor, resulta en menos efectos cognitivos. Estos aparatos incorporan la posibilidad de modificar parámetros y hacer autotest. También suele incorporarse monitorización del electrocardiograma y electroencefalograma (1).

\section{Estrategias de dosificación: titulación empírica}

En la primera sesión se realiza la titulación, que se inicia con la aplicación de una dosis subconvulsiva. Una vez establecida la dosis del umbral convulsivo se aplicará el porcentaje correspondiente adecuado según la colocación de los electrodos (16).

\section{Estrategias de dosificación: fórmula ajustada a factores}

Los parámetros del estímulo se ajustan en función de la edad, la colocación de electrodos y el sexo (Tabla 4) (20).

TABLA 4. ESTRATEGIAS DE DOSIFICACIÓN

\begin{tabular}{|l|c|c|c|c|}
\hline & $\begin{array}{c}\text { Amplitud } \\
\text { de pulsos }\end{array}$ & $\begin{array}{c}\text { Frecuencia } \\
\text { de pulsos }\end{array}$ & $\begin{array}{c}\text { Duración } \\
\text { del estímulo }\end{array}$ & $\begin{array}{c}\text { Intensidad } \\
\text { de la corriente }\end{array}$ \\
\hline Unilateral mujer & 1,2 & 60 & 1,25 & 0,8 \\
\hline Unilateral hombre & 1,2 & 80 & 1,25 & 0,8 \\
\hline Bilateral mujer & 1,4 & 80 & 1,25 & 0,8 \\
\hline Bilateral hombre & 1,4 & 90 & 1,5 & \\
\hline $\begin{array}{l}\text { Edad: } \\
\text { <30 años. Disminuir una posición de frecuencia o duración. } \\
\text { >60 años. Aumentar una posición de frecuencia o duración. }\end{array}$
\end{tabular}

\section{Aplicación de la TEC por umbral convulsivo}

El umbral convulsivo se define como la mínima dosificación eléctrica necesaria para generar una convulsión tónica generalizada (18). 
Para el $40 \%$ de los pacientes el umbral convulsivo promedio en TEC unilateral es de $50 \mathrm{mC}$. Luego, se recomienda ir disminuyendo a $25 \mathrm{mC}$ en el caso de pacientes con medicación que disminuya el umbral convulsivo.

Se consideran como estímulos efectivos aquellos que sean mayores a 50 a $150 \%$ del umbral convulsivo de base. Por lo que, una vez alcanzado el umbral convulsivo, se debe elevar la corriente eléctrica un $50 \%$ de ese umbral.

Si se falla a determinado estimulo, el siguiente debe ser de una intensidad de 50-100\% superior (18). Si no se obtiene el estímulo umbral se debe considerar la revisión de la medicación así como la oxigenación y la hidratación (20).

\section{Modificación de la estimulación}

El umbral convulsivo puede cambiar a lo largo del tratamiento, por lo que puede resultar necesario reajustar la dosis del estímulo. A más, en caso de respuesta clínica lenta o insuficiente se tiene que considerar un incremento de la dosis del estímulo.

La dosis del estímulo se aumenta incrementando primero la frecuencia de los pulsos, después la duración, la amplitud y finalmente la intensidad de la corriente (16).

\section{Colocación de electrodos}

Los electrodos pueden situarse bifrontotemporal, bifrontal y unilateral derecha. Se colocan a los costados de la cabeza y el punto medio de cada electrodo se sitúa a $2,5 \mathrm{~cm}$ por sobre el punto medio de la línea imaginaria que une el trago con el canto externo del ojo (16).

Se debe preferir TEC bilateral cuando el procedimiento sea mayor a diez sesiones o cuando no se pueda establecer la dominancia del paciente. Se recomienda que en TEC bilateral se dé entre 50 a $150 \%$ de la carga eléctrica necesaria para sobrepasar el umbral convulsivo (16). El tiempo de recuperación promedio para este estímulo es de 20 minutos (18).

Se recomienda TEC unilateral si existe deterioro cognitivo previo. En caso de la TEC unilateral la carga eléctrica administrada será de $150 \%$ a $500 \%$ la carga para sobrepasar el umbral convulsivo. El tiempo de recuperación promedio es de 30 minutos (18). Para la colocación unilateral derecha se coloca un electrodo en posición frontotemporal y el otro a la derecha del vértice, en la región centroparietal (15).

\section{Convulsiones}

Para que el estímulo eléctrico se considere efectivo debe generar un estímulo físico suficiente para provocar una convulsión que dure no menos de 15 segundos por observación visual y 25 segundos por registro electroencefalográfico (18).

Toda actividad convulsiva de más de 3 minutos se considera "convulsión prologada" y deberá abortarse por el riesgo elevado de confusión y deterioro cognitivo. 


\section{Crisis fallidas}

Se aconseja re-estimular a mayor intensidad (más de 50\%-100\%). Se pueden hacer hasta 4-5 estimulaciones. Entre cada estimulación deben trascurrir 20 segundos. Puede resultar necesario volver a administrar agentes anestésicos y/o relajantes.

Se consideran métodos de potenciación de crisis: Reducir dosis de anestesia y medicaciones con acciones anticonvulsivantes, utilizar flumazenilo para revertir la acción benzodiazepínica. Proporcionar hiperventilación enérgica antes de la crisis. Utilizar anestésicos con menos efectos sobre el umbral convulsivo (ketamina o etomidato).

\section{Tiempo y número de sesiones}

Como mínimo se recomiendan entre 6 a 12 sesiones. La frecuencia de sesiones debe ser de 2 a 3 veces por semana (20). En los casos en los que los pacientes no presenten mejoría alguna después de 6 sesiones de TEC, se debe suspender la terapia y se declara "fallo terapéutico". Si hay respuesta, se recomienda el uso de 6 sesiones adicionales. No se recomienda preestablecer el número de sesiones para ninguno de los pacientes dado que éste depende de la respuesta individualizada.

\section{EFECTOS SECUNDARIOS}

La tabla 5 informa de los efectos secundarios más frecuentemente observados luego de la aplicación de TEC.

\section{TABLA 5. EFECTOS SECUNDARIOS DE LA TEC}

\begin{tabular}{|c|c|}
\hline Confusión & $\begin{array}{l}\text { La confusión puede durar desde minutos hasta horas (1). Además, existe la } \\
\text { posibilidad que los pacientes desarrollen delirium. Se debe vigilar si el pa- } \\
\text { ciente presenta estados de agitación, intranquilidad o agresión luego de las } \\
\text { primeras sesiones y se recomienda el uso de benzodiazepinas en dosis altas } \\
\text { (20). }\end{array}$ \\
\hline Deterioro cognitivo & $\begin{array}{l}\text { El médico debe evaluar la orientación y la memoria antes y después de la TEC. } \\
\text { Aunque se pueden observar cambios en la memoria, en la atención y en la } \\
\text { concentración en los días posteriores al TEC, los mismos se normalizan (19). } \\
\text { Se sugiere, que si se sospechase deterioro cognitivo por TEC se cambie el } \\
\text { pulso desde onda sinusal a breve, disminuir la intensidad del estímulo, pro- } \\
\text { longar el intervalo entre sesiones y reducir los agentes anestésicos (20). La } \\
\text { hiperoxigenación pre-TEC y la TEC unilateral también han reducido muy sig- } \\
\text { nificativamente este tipo de efectos indeseables (1). }\end{array}$ \\
\hline Cefalea & La cefalea puede ser prevenida con analgésicos $(1,20)$. \\
\hline Estatus epiléptico & $\begin{array}{l}\text { Hay un riesgo potencialmente mayor de convulsiones post TEC hasta las } 24 \\
\text { horas posteriores al procedimiento en el caso de los adolescentes. Para el } \\
\text { manejo del estatus epiléptico se recomienda dar diazepam, lorazepam o met- } \\
\text { hohexital. }\end{array}$ \\
\hline Amnesia & $\begin{array}{l}\text { La TEC puede generar amnesia anterógrada. También se puede constatar am- } \\
\text { nesia retrógrada especialmente sobre datos autobiográficos cuando se aplica }\end{array}$ \\
\hline
\end{tabular}


TEC bilateral. El grado de amnesia no se correlaciona con la mejoría terapéutica y en general las pérdidas de memoria mejoran durante el curso de la TEC (20).

\begin{tabular}{|c|l|}
\hline Apnea prolongada & $\begin{array}{l}\text { El empleo de relajantes musculares se relaciona con la aparición, también in- } \\
\text { frecuente, de apnea prolongada }(1,16) .\end{array}$ \\
\hline Mialgias & $\begin{array}{l}\text { Frecuentes, debido a la propia convulsión y a la administración de succinilco- } \\
\text { lina }(25) \text {. Las fracturas y las luxaciones (especialmente mandibulares) son } \\
\text { prácticamente inexistentes con la utilización de relajantes musculares y pro- } \\
\text { tección bucal (1). }\end{array}$
\end{tabular}

\section{VALORACIÓN POST TEC}

Mientras el paciente se recupera de la anestesia lo primero será asegurarse de que tiene una buena ventilación y oxigenación. Deben monitorizarse el pulso y la tensión arterial hasta que sean estables y el paciente se encuentre vigil y orientado.

Si se prevé riesgo para el paciente pues este puede caerse o golpearse, puede estar indicado aplicar medidas físicas de contención. El midazolam endovenoso es útil para sedar al pequeño porcentaje de pacientes que se agita durante la recuperación.

\section{TEC EN RÉGIMEN AGUDO, DE CONTINUACIÓN Y DE MANTENIMIENTO}

\section{TEC agudo}

Se realiza en pacientes ingresados en Unidades Psiquiátricas o en forma ambulatoria, con aplicación de 2 o 3 electroconvulsiones por semana y de 8 a 12 como media para completar un tratamiento (2).

\section{TEC de continuación}

Se define como TEC de continuación cuando se continúa la terapia por 6 meses más para consolidar un buen resultado de un tratamiento agudo y evitar recaídas (26). Las recaídas durante la TEC de continuación usualmente responden rápidamente a la TEC con el incremento de la frecuencia de las sesiones (2).

\section{TEC DE MANTENIMIENTO}

Esta modalidad es definida como un régimen a largo plazo, en el que los pacientes reciben TEC en intervalos constantes o variables de tiempo.

El programa consiste en sesiones semanales, seguidas de otras quincenales, pasando después a su intervalo mensual, hasta que se considera que existe una adecuada estabilidad al cabo de 6 o 7 meses. Su objetivo es evitar recurrencias $(2,26)$.

No existe consenso sobre la duración de la TEC de mantenimiento, pero debe ser reevaluado cada 6 meses y considerar que en pacientes refractarios al tratamiento puede ser mantenida a lo largo de la vida, los que son los pacientes TEC-dependientes. 
La administración de TEC ambulatoria y de mantenimiento sigue el modelo de cirugía ambulatoria. El paciente debe acudir al Hospital en la mañana y puede ser dado de alta unas horas tras el tratamiento, bajo la tutela de un adulto responsable, recomendándose no conducir vehículos ni realizar tareas que requieren elevada atención (2).

La TEC de continuación y la de mantenimiento reducen costos en cuanto a medicación e internación y permiten al paciente aumentar su productividad y días de trabajo (27-29).

\section{CONCLUSIÓN}

Desde la introducción de la TEC, la técnica ha experimentado muchos cambios. Actualmente se utilizan aparatos de electroconvulsión de pulso breve, mejor monitorización de los pacientes, utilización de anestesia y una indicación más selectiva de las patologías psiquiátricas que responden a este tratamiento; todos estos avances hacen que la TEC actualmente continúe en plena vigencia y siga siendo la terapia de primera elección en varias patologías y de segunda elección en otras que no mejoran con el manejo psicofarmacológico.

Un comité de TEC debe examinar todos los casos que requieran TEC y autorizar su realización teniendo en cuenta sus indicaciones, contraindicaciones y otras condiciones médicas que podría suponer un riesgo. Este comité, es el responsable que siempre se actúe respetando los principios bioéticos, sin violar los derechos humanos de los pacientes.

Existen aún factores que alientan la estigmatización de la TEC, al requerir el uso de corriente eléctrica y provocar convulsiones, existe el temor trasmitido por informes inexactos de ocasionar lesiones cerebrales. Es importante implementar programas educativos que favorezcan el conocimiento científico de esta técnica, promoviendo la investigación y la experiencia a fin de mantener el compromiso del médico de actualizar constantemente su conocimiento para ofrecer tratamientos seguros, accesibles y eficaces.

Se espera que este protocolo se constituya en un instrumento útil para la aplicación de la TEC, procedimiento que podría modificar el curso de la enfermedad, no sólo en cuanto a su sintomatología, sino que también en cuanto a la mejoraría en el perfil de efectos adversos, costes económicos, disminución en el impacto de las recaídas, rehospitalizaciones y mejoraría en la calidad de vida de los paciente y de sus familias.

\section{CONFLICTOS DE INTERÉS Y FUENTE DE FINANCIACIÓN}

La autora declara no poseer conflictos de interés. Fuente de financiación: ninguna.

\section{AGRADECIMIENTOS}

La autora agradece al Prof. Dr. Martín Moreno, Profesor Adjunto de Psiquiatría de la Facultad de Ciencia Médicas de la Universidad Nacional de Asunción (Paraguay), por su contribución teórica e interpretativa a esta revisión.

\section{REFERENCIAS BIBLIOGRÁFICAS}

1. Bernardo M, Cervera S. Consenso Español sobre la Terapia Electroconvulsiva. Barcelona: Sociedad Española de Psiquiatría; 1999. URL. 
2. Medina L, López M. Unidad de TEC ambulatoria y de Mantenimiento con Carácter Regional. Murcia: Hospital Psiquiátrico Román Alberca; 2011. URL.

3. Kaplan H. Sinopsis de Psiquiatría. 10ª ed. New York: McGraw Hill; 2009.

4. Bollwing T. How does the electroconvulsive Therapy work? Theories of its mechanism. Can J Psychiatry. 2011;56(1):13-18. http://dx.doi.org/10.1177/070674371105600104

5. Ríos $B$, Vicente $N$. Mecanismo de acción de la terapia electroconvulsiva en la depresión. Actas Esp Psiquiatr 2001;29(3):199-207. URL.

6. Merkl A, Heuser I, Bajbouj M. Antidepressant Electroconvulsive Therapy: Mechanism of action, recent advances and limitations. Exp Neurol. 2009;219(1):20-26. http://dx.doi.org/10.1016/j.expneurol.2009.04.027

7. Sanz F, Tejadas A. Terapia electroconvulsiva en la Enfermedad de Parkinson. Psiquiatría Biológica. 1995;2(6):11-22.

8. Bernardo M. Terapéuticas Biológicas en Psiquiatría. En: Soler-Insa PA, Gascón J. Recomendaciones Terapéuticas en los Trastornos Mentales. Comité de Consenso de Catalunya en Terapéutica de los Trastornos Mentales. 3a ed. Barcelona: Ars Médica; 2005. p. 519-526.

9. Birkenhäger TK, van den Broek WW, Mulder PG, de Lely A. One year outcome of Pshycotic Depression After Successful Electroconvulsive Therapy. J ECT. 2005;21(4):221226. URL.

10. Tharyan P, Adams C, Electroconvulsive Therapy for Schizophrenia. Cochrane Database Syst Rev. 2002;(2):CD000076. URL.

11. Asociación Psiquiátrica de América Latina y Asociación Mexicana de Psiquiatría. Guía basada en la evidencia de la Asociación Psiquiátrica de América Latina y de la Asociación Mexicana para el tratamiento del paciente con esquizofrenia. Salud Mental 2014;37(1):63-64. URL.

12. Parellada E, Fernández E. Esquizofrenia, del caos mental a la esperanza. 1a ed. BarceIona: Morales y Torres; 2004.

13. National Institute on Clinical Excellence (NICE). Guidance on the use of Electroconvulsive Therapy. London: National Institute on Clinical Excellence; 2010. URL.

14. Braga RJ, Petrides G. The combined use of electroconvulsive therapy and antipsychotics in patients with schizophrenia J ECT. 2005;21(2):75-83. URL.

15. Mankad MV, Beyer J. Clinical Manual of electroconvulsive Therapy. New York: American Psychiatric Publising; 2010.

16. Pons A. Protocol TEC. Programa de TEC Clínic, Recomenacions I procediments d'actuació. Barcelona: Institut Català de la Salu; 2001.

17. Castro R, Pérez I. Normas para la Terapia Electroconvulsiva. Sala de TEC y Recuperación. San José: Hospital Nacional Psiquiátrico de Costa Rica; 2009.

18. Royal College of Psychiatrists. The ECT Handbook: The Third Report of the Royal College of Psychiatrist Special Committee on ECT. London: The Royal College of Psychiatrists; 2005.

19. McClellan J, Kowatch R, Findling RL. Practice parameter for the Assessment and Treatment of Children and Adolescents with Bipolar Disorder J Am Acad Child Adolesc Psychiatry. 2007;46(1):107-25. http://dx.doi.org/10.1097/01.chi.0000242240.69678.c4

20. American Psychiatric Association. The practice of Electroconvulsive Therapy. Recommendations for Treatment, Training and Privileging. 2nd ed. Washington: American Psychiatric Association; 2001. 
21. Stek ML, Wurff van der FFB, Hoogendijk WJG, Beekman ATF. Electroconvulsive therapy for the depressed elderly. Cochrane Database of Systematic Reviews 2003; 2: CD003593.

22. Sackeim HA, Dillingham EM, Prudic J, Cooper T, McCall WV, Rosenquist $P$, et al. Effect of concomitant pharmacotherapy on electroconvulsive therapy outcomes: short-term efficacy and adverse effects. Arch Gen Psychiatry. 2009;66(7):729-737. http://.dx.doi.org/10.1001/archgenpsychiatry.2009.75

23. Painuly N, Chakrabarti S. Combined use of electroconvulsive therapy and antipsychotics in schizophrenia: the Indian evidence. A review and a meta-analysis. J ECT. 2006 Mar;22(1):59-66. URL.

24. Vidal M. La Problemática del Consentimiento Informado. Cuadernos de Bioética 1998;1:12-24. URL.

25. Simón Lorda D, Simón Lorda P, Rodríguez López A, Concheiro Carro L. El consentimiento Informado en Psiquiatría: un formulario escrito de consentimiento para terapia electroconvulsiva (TEC) o electroshock. An Psiquiatr 1992;8(7):245-252. URL.

26. Rabheru K1, Persad E. A Review of Continuation and Maintenance Electroconvulsive Therapy. Can J Psychiatry 1997;42:476-484. URL.

27. Fox HA. Continuation and Maintenance Electroconvulsive Therapy-A Conceptual Framework? http://dx.doi.org/10.1097/YCT.0000000000000224.

28. Petrides G, Tobias KG, Kellner CH, Rudorfer MV. Continuation and Maintenance Electroconvulsive Therapy for Mood Disorders: Review of the Literature. Neuropsychobiology. 2011;64(3):129-140. http://.dx.doi.org/10.1159/000328943

29. Martínez-Amorós E, Cardoner N, Gálvez V, Urretavizcaya M. Eficacia y patrón de uso de la terapia electroconvulsiva de continuación y mantenimiento en el trastorno depresivo mayor. Rev Psiquiatr Salud Ment 2012;5:241-253. http://dx.doi.org/10.1016/j.rpsm.2012.06.004 